\title{
Plasmon-Induced Magneto-Optical Activity in Nanosized Gold Disks
}

\author{
B. Sepúlveda, ${ }^{1}$ J. B. González-Díaz, ${ }^{2}$ A. García-Martín, ${ }^{2}$ L. M. Lechuga, ${ }^{1}$ and G. Armelles ${ }^{2}, *$ \\ ${ }^{1}$ Research Center on Nanoscience and Nanotechnology-CIN2 (CSIC) and CIBER-BBN, 08196 Bellaterra, Spain \\ ${ }^{2}$ IMM-Instituto de Microelectrónica de Madrid (CNM-CSIC), Isaac Newton 8, PTM, E-28760 Tres Cantos, Madrid, Spain
}

(Received 14 October 2009; published 7 April 2010)

In this Letter we show that nanostructures made out of pure noble metals can exhibit measurable magneto-optic activity at low magnetic fields. This phenomenon occurs when the localized surface plasmon resonance of the nanostructure is excited in the presence of a static magnetic field parallel to the propagation of incident light. The large magneto-optical response observed comes from an increase of the magnetic Lorentz force induced by the large collective movement of the conduction electrons in the nanostructures when the resonance is excited.

DOI: 10.1103/PhysRevLett.104.147401

Nanoplasmonics has attracted a great amount of interest in the past decade due to the ability of surface plasmons to surpass the diffraction limit of conventional optics, opening paths to envisage novel nanophotonic devices [1,2]. This is possible thanks to the ability to localize the electromagnetic field in very small volumes. This property has also been used to alter the magneto-optical (MO) response in nanostructures made out of noble metals and ferromagnets [3-9]. In these systems the modification of the electromagnetic field due to plasmon excitation has boosted the MO response of the composite system: the ferromagnet induces the MO activity, and the noble metal allows the excitation of nondamped plasmons which enhance the electromagnetic field intensity inside the ferromagnet and, therefore, the MO response of the system. On the other hand, the magneto-optical activity in noble metals is much weaker than that of ferromagnetic materials for reasonable magnetic fields $(0.5-1 \mathrm{~T})$, and in order to obtain a $\mathrm{MO}$ activity in pure noble metals films similar to those in ferromagnetic layers, a magnetic field of several hundreds of Tesla should be applied. This is even more critical where nanostructures come into play since the reduction of "active" material would make the signal drop significantly. However, the excitation of localized surface plasmon resonances (LSPRs) might exert a positive role. In this Letter we show for the first time that plasmonic nanostructures made out of pure gold alone can exhibit a sizable MO activity at low magnetic fields (of the order of $1 \mathrm{~T}$ ). This effect is due to the large enhancement of the magnetic Lorentz force induced by the collective movement of the conduction electrons when the LSPR is excited. To understand the physical mechanism of the effect, we first give an analytical description of the polarizability of a metal nanoparticle in the presence of a static magnetic field, followed by the experimental demonstration of the MO effects.

It is well known that a moving charge $q$ with speed $\boldsymbol{v}$ in the presence of a magnetic field $B$ experiences a Lorentz force $\left(F_{\text {Lor }}\right)$, which is directed perpendicularly to the magnetic field and the trajectory of the moving charge, i.e., $\mathbf{F}_{\text {Lor }}=q(\mathbf{v} \times \mathbf{B})$. Such Lorentz force can also be induced
PACS numbers: 78.67.Bf, 73.20.Mf, 78.20.Ls, 78.68.+m

in oscillating dipoles due to their associated moving charges [10]:

$$
\mathbf{F}_{\text {Lor }}=\frac{d \mathbf{p}}{d t} \times \mathbf{B} .
$$

According to Eq. (1), the applied magnetic field exerts a torque in the variable electric dipole when the magnetic field is perpendicular to it. This equation suggests that every material illuminated with a light beam is susceptible to undergo a Lorentz force since:

$$
\mathbf{F}_{\text {Lor }}=i \omega \alpha \mathbf{E}_{0} \times \mathbf{B},
$$

where $\alpha$ is the polarizability of the material, $\mathbf{E}_{\mathbf{0}}$ is the electric field amplitude of the incident the light beam, and $\omega$ is its frequency. However, this effect is generally very weak unless either the external magnetic field or the polarizability is very strong. Taking advantage of later property, we are going to show how the large enhancement of the polarizability in plasmonic nanostructures is enough for the generation of MO effects at moderate intensity magnetic fields, without the need of ferromagnetic materials.

For simplicity, we first consider the polarizability of a spherical metal nanoparticle of radius $a$, much smaller than light wavelength $\lambda$, which can be approximated by a quasistatic model. In the presence of a magnetic field, the polarizability of the nanoparticle becomes anisotropic and can be described by the following tensor: $\tilde{\boldsymbol{\alpha}}=4 \pi a^{3}(\tilde{\boldsymbol{\varepsilon}}-$ $\left.\varepsilon_{d} \tilde{\mathbf{1}}\right)\left(\tilde{\boldsymbol{\varepsilon}}+2 \varepsilon_{d} \tilde{\mathbf{1}}\right)^{-1}$, where $\tilde{\mathbf{1}}$ represents the identity $3 \times 3$ matrix, $\tilde{\boldsymbol{\varepsilon}}$ the dielectric tensor of the metal, and $\varepsilon_{d}$ the dielectric constant of the external dielectric medium [11]. If we describe the optical properties of the metal by a free electron Drude model and the external magnetic field is applied in the $z$ direction, the components of $\tilde{\boldsymbol{\varepsilon}}$ are given by

$$
\begin{aligned}
& \varepsilon_{x x} \approx \varepsilon_{y y} \approx \varepsilon_{z z} \approx \varepsilon_{\infty}-\varepsilon(\omega), \\
& \varepsilon_{x y} \approx i \varepsilon(\omega) \frac{\omega_{c}}{\omega+i \gamma},
\end{aligned}
$$

where $\varepsilon(\omega)=\varepsilon_{\infty} \omega_{p}^{2} / \omega(\omega+i \gamma)$, where $\omega_{p}$ is the electron gas plasma frequency, $\gamma$ is the electronic relaxation 
constant, and $\omega_{c}$ is the cyclotron frequency. In this expression we have neglected higher order terms in $\omega_{c}$. The nondiagonal MO term $\varepsilon_{x y}$ arises as a consequence of the induced transversal movement of the conduction electrons by the external magnetic field. In plasmonic materials made out of noble metals $\omega_{c} \ll \omega_{p}$ and, therefore, the MO effects are generally very weak [12], which is manifested in almost 3 orders of magnitude smaller amplitude of the MO constant with respect to Co in the visible (at $700 \mathrm{~nm}, \varepsilon_{x y}=0.8+i 0.16$ for Co, whereas $\varepsilon_{x y}=10^{-4}+$ $i 10^{-3}$ for Au at $1 \mathrm{~T}$ ). However, the optical properties of plasmonic materials can be drastically modified when they are nanostructured, due to their LSPR, which is reflected in their polarizability tensor:

$$
\begin{aligned}
& \alpha_{x x} \approx 4 \pi a^{3} \frac{\varepsilon_{x x}-\varepsilon_{d}}{\varepsilon_{x x}+2 \varepsilon_{d}}, \\
& \alpha_{x y} \approx 4 \pi a^{3} \frac{3 \varepsilon_{x y} \varepsilon_{d}}{\left(\varepsilon_{x x}+2 \varepsilon_{d}\right)^{2}}=\frac{3 \varepsilon_{x y} \varepsilon_{d} \alpha_{x x}^{2}}{4 \pi a^{3}\left(\varepsilon_{x x}-\varepsilon_{d}\right)^{2}} .
\end{aligned}
$$

Equation (4) shows that the LSPR is excited when $\varepsilon_{x x}=$ $-2 \varepsilon_{d}$ and, interestingly, predicts the generation of an electric dipole transversal to that excited by the induced light, which is proportional to the magnetic field amplitude, and exhibits the same resonant condition. Therefore, in the presence of an external magnetic field, the net induced dipole in the nanoparticle oscillates at an angle $\theta_{K}$ with respect to the electric field of incident light [see Fig. 1(a)], given by

$$
\theta_{K}=\arctan \left(\frac{\alpha_{x y}}{\alpha_{x x}}\right) \approx \frac{3 \varepsilon_{d} \varepsilon_{x y} \alpha_{x x}}{4 \pi a^{3}\left(\varepsilon_{x x}-\varepsilon_{d}\right)^{2}} .
$$

The angular shift of the induced electric dipole is proportional to the polarizability of the particle and, therefore, will be maximized when the LSPR condition is fulfilled.
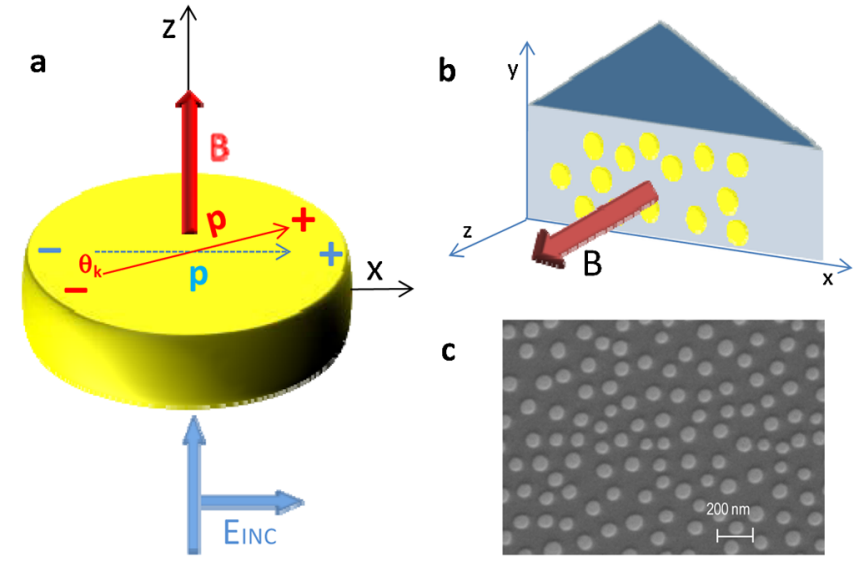

FIG. 1 (color online). (a) Schematic of the MO effect induced by the Lorentz force in a metal nanoparticle. (b) Magneto-optic Kerr polar experimental configuration. (c) SEM images of the gold nanodisk arrays.
This dependence is consistent with the creation of a Lorentz force proportional to the polarizability of the nanoparticle [see Eq. (2)]. As a consequence of the angular shift, the polarization of the scattered light by the nanoparticles in the far field will present the same shift. Since $\theta_{K}$ is a complex number, we expect two effects in the scattered photons: a rotation of their polarization (real part of $\theta_{K}$ ) and a dephasing or change in the ellipticity state with respect to incident photons (imaginary part of $\theta_{K}$ ), and both effects will be amplified at the LSPR wavelength. As the resonant wavelength is highly dependent on the size, shape, and composition of the nanostructures, we expect the MO activity to exhibit the same tunability as the optical properties of plasmonic nanostructures.

To experimentally probe the plasmonic MO effects we have patterned arrays of nanodisks with different aspect ratios in glass substrates. These arrays, fabricated by a large scale colloidal lithography technique [13], are composed of a random distribution of nanodisks with subwavelength separation distances (Fig. 1), to avoid diffraction effects. In particular, we have fabricated arrays of gold nanodisks with aspect ratios of 2.2 and 6 (diameters $D=$ 70 and $120 \mathrm{~nm}$ and height $h=32$ and 20, respectively), where we have kept similar volume fraction of nanostructures, around $10 \%$.

Samples have been optically characterized by extinction spectroscopy, while the MO effects in the backscattered light have been probed in a MO Kerr setup [14] in normal incidence, in which a magnetic field of $0.8 \mathrm{~T}$ is applied normal to the arrays [see Fig. 1(b)]. In these measurements we simultaneously extract the rotation of the polarization and the change of the ellipticity induced by the magnetic field in the reflected photons. To minimize the MO effects associated to the glass substrates, these samples have been attached to a glass prism via refractive index matching oil [see Fig. 1(b)].

The experimental results of the arrays of gold nanodisks on diamagnetic borosilicate glass (D262-M) substrates are gathered in Fig. 2. As it has been theoretically predicted above, a substantial MO activity appears at the same spectral position as the extinction peaks associated to the LSPR, showing linear dependence on the applied magnetic field (Fig. 2). In addition, both extinction and MO activity exhibit similar redshifts when the aspect ratio of the nanodisks increases, showing the LSPR connection with both optic and magneto-optic effects. Another link between the MO activity and the resonant polarizability of the nanostructures is found in the increase of the MO effects when the resonance redshifts, as a consequence of a stronger dipole in larger nanostructures and a weaker damping due to the separation of the resonance from interband transitions of gold. On the other hand, the shape of rotation and ellipticity spectra are directly related to the resonant shapes of the imaginary and real parts of the polarizability, respectively, as can be deduced from Eq. (5). In order to 


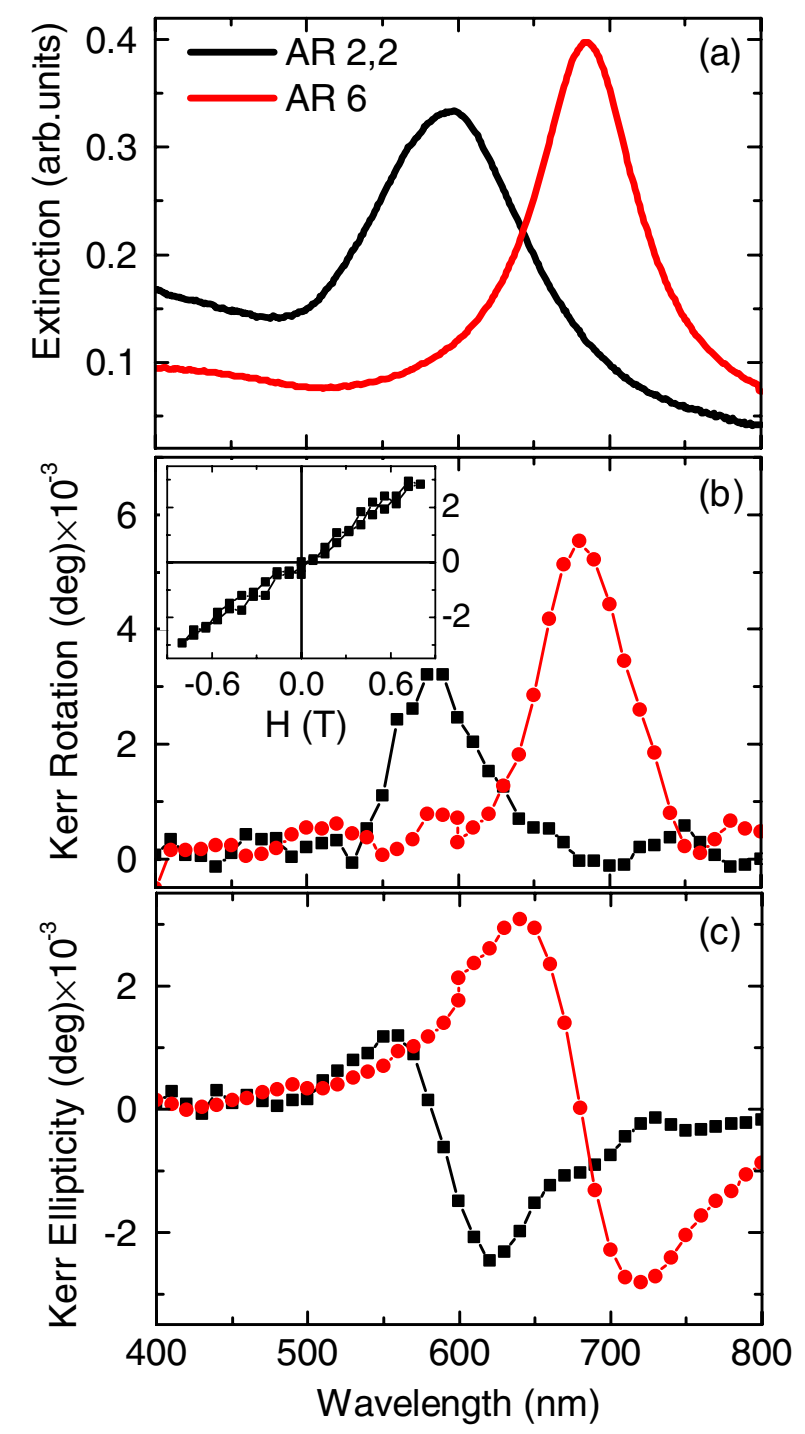

FIG. 2 (color online). Experimental optical and magnetooptical effects in Au nanodisk arrays: (a) optical extinction, (b) Kerr rotation, and (c) ellipticity of nanodisk arrays with aspect ratios (AR) $2.2(D=70, h=32)$ (squares) and $6(D=$ 120, $h=20$ ) (circles). The inset in (b) represents the Kerr rotation variation as a function of the magnetic field for the $\mathrm{Au}$ nanodisks with aspect ratio 2.2.

unambiguously demonstrate that the origin of the MO effect comes from the metal nanoparticles, we have compared these results to those from nanoparticles fabricated on paramagnetic glass (MOS105), whose Verdet constant has sixfold larger amplitude and opposite sign. Despite the large difference in the Verdet constants, the observed $\mathrm{MO}$ effects are similar to those of Fig. 2, especially in the sign of rotation and ellipticity, which excludes any noticeable influence of the MO activity of the glass substrate on the observed MO effects. It is worth noticing that we have observed similar results in other noble metals as silver; therefore, this effect can be seen as general for Drude-like metals.
To validate the hypothesis of the MO activity caused by a LSPR amplification of the magnetic Lorentz force, we compare the experimental results to two different theoretical models: a Maxwell-Garnett effective medium model [15], suitable for low concentration assemblies of randomly distributed nanoparticles, resembling the experimental arrays, and a scattering matrix formalism, valid for ordered arrays of nanoparticles [16]. In both calculations the nanoplasmonic medium is placed over a glass substrate, the incident medium is air, and we assume a modified Drude model for gold, to include the effect of interband transitions [17] and an external magnetic field of $0.8 \mathrm{~T}\left(\omega_{c}=1.41 \times 10^{11} \mathrm{~s}^{-1}\right)$. In Maxwell-Garnett we consider arrays of oblate ellipsoidal nanoparticles with the same aspect ratio and volume concentration as those of the experiments, and a dielectric constant of the environment $\varepsilon_{d}=1.69$, to compensate the effect of the substrate on the peak position of the nanoparticles, whereas in the scattering matrix we employ a square array of disk-shaped nanoparticles with analogous geometrical parameters as the experiments.

These calculations predict very similar features to those experimentally found for the arrays of gold nanodisks (see

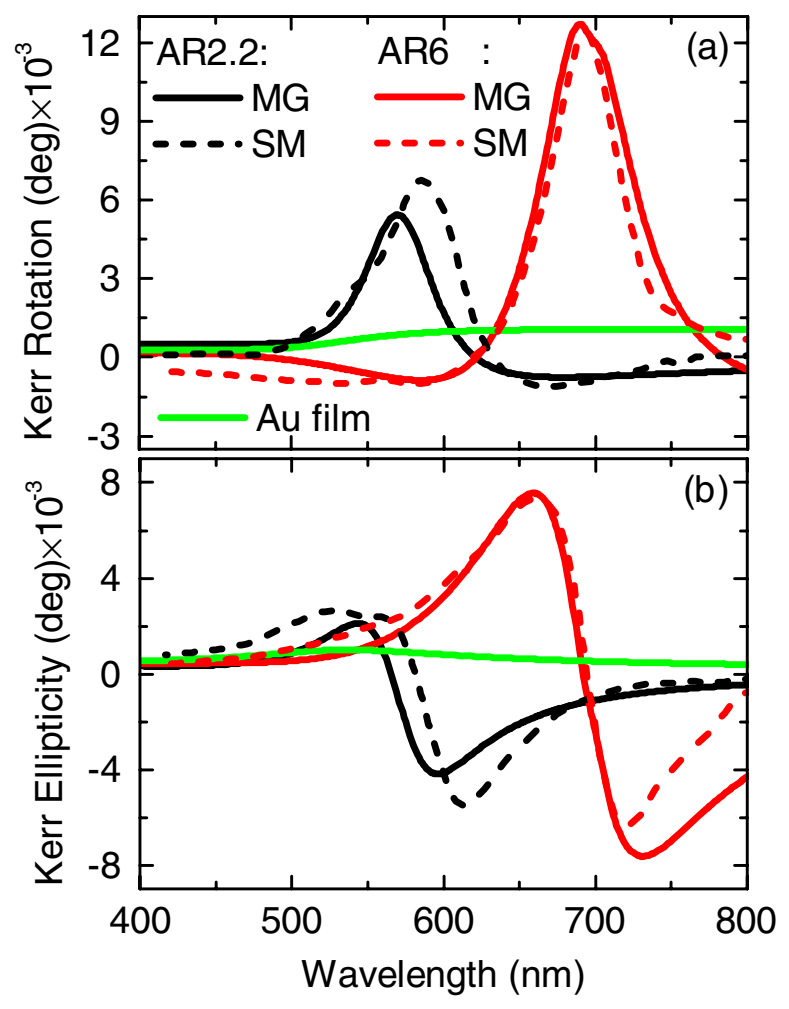

FIG. 3 (color online). Theoretical Kerr rotation (top panel) and ellipticity (bottom panel) in gold nanoparticles and films. Comparison of the Maxwell-Garnett (MG) model for arrays of randomly distributed nanoparticles [dark gray (red) lines and black lines), the scattering matrix (SM) model for ordered arrays of nanoparticles (symbols), and the MO effects of a continuous $20 \mathrm{~nm} \mathrm{Au}$ film [light gray (green) line]. 
Fig. 3), i.e., a resonant behavior in the rotation and ellipticity whose spectral position redshifts for larger aspect ratios, and similar shapes for rotation and ellipticity spectra as those of the imaginary and real parts of the polarizability, respectively. However, the amplitude of the MO effect in the theoretical models is slightly larger than the experimental values, mainly due to the higher electronic damping in the experimental nanostructures caused by dephasing effects and surface roughness. It is clear from these results that, at these concentrations, order plays an insignificant role in the MO activity due to the large separation distances between particles, where near-field or diffractive couplings are weak. Indeed, both theoretical models converge to the solution of a single nanoparticle [Eq. (5)] when the substrate and incident media have the same refractive index and the volume concentration of nanoparticles is reduced below $1 \%$. The contrast of these MO effects to those expected for a $20 \mathrm{~nm}$ thick gold film highlights the LSPR amplifying behavior (Fig. 3), which is larger than 1 order of magnitude in the nanostructured media, even though the quantity of material is 10 times smaller. Indeed, the observed Kerr rotation and ellipticity is only 20 -fold lower than that of $\mathrm{Au} / \mathrm{Co} / \mathrm{Au}$ nanostructures of Ref. [7] despite the 800 times smaller MO constant in gold than in cobalt, which underlines the different origin of the MO activity in both systems.

In conclusion, we have experimentally demonstrated that plasmonic nanostructures can exhibit a substantial MO activity. This effect is due to the increase of the magnetic Lorentz force induced by the large collective movement of the conduction electrons in the nanostructures when the LSPR is excited. To understand the physical mechanism of the effect, we have relied on a simple approach based on the polarizability of a metal nanoparticle in the presence of a static magnetic field.

Financial support from EU NMP3-SL-2008-214107 (Nanomagma), CAM S2009/TIC-1476 (MICROSERES), M. Botin Foundation, EU MC2Access program, and
MICINN ("FUNCOAT" CONSOLIDER INGENIO 2010 CSD2008-00023, MAT2008-06765-C02-01/NAN, NAN2004-09195-C04-01, and "Ramon y Cajal" program) is gratefully acknowledged.

*gaspar@imm.cnm.csic.es

[1] W. L. Barnes, A. Dereux, and T.W. Ebbesen, Nature (London) 424, 824 (2003).

[2] H. A. Atwater, Sci. Am. 296, No. 4, 56 (2007).

[3] V. I. Safarov, V. A. Kosobukin, C. Hermann, G. Lampel, J. Peretti, and C. Marliere, Phys. Rev. Lett. 73, 3584 (1994).

[4] V. I. Belotelov, L. L. Doskolovich, and A. K. Zvezdin, Phys. Rev. Lett. 98, 077401 (2007).

[5] Y.M. Strelniker and D.J. Bergman, Phys. Rev. B 77, 205113 (2008).

[6] G. A. Wurtz, W. Hendren, R. Pollard, R. Atkinson, L. Le Guyader, A. Kirilyuk, Th. Rasing, I. I. Smolyaninov, and A. V. Zayats, New J. Phys. 10, 105012 (2008).

[7] J. B. Gonzalez-Diaz et al., Small 4, 202 (2008).

[8] P. K. Jain, Y.H. Xiao, R. Walsworth, and A.E. Cohen, Nano Lett. 9, 1644 (2009).

[9] C. S. Levin et al., ACS Nano 3, 1379 (2009).

[10] D. Bedeaux and P. Mazur, Physica (Amsterdam) 67, 23 (1973).

[11] D. A. Smith and K. L. Stokes, Opt. Express 14, 5746 (2006).

[12] G. Duchs, G. Rikken, T. Grenet, and P. Wyder, Phys. Rev. Lett. 87, 127402 (2001).

[13] H. Fredriksson et al., Adv. Mater. 19, 4297 (2007).

[14] W.S. Kim, M. Aderholz, and W. Kleemann, Meas. Sci. Technol. 4, 1275 (1993).

[15] C.F. Bohren and D. R. Huffman, Absorption and Scattering by Small Particles (Wiley-Interscience, New York, 1983).

[16] A. Garcia-Martin, G. Armelles, and S. Pereira, Phys. Rev. B 71, 205116 (2005).

[17] P. G. Etchegoin, E. C. Le Ru, and M. Meyer, J. Chem. Phys. 125, 164705 (2006). 